\title{
Fratricide: Defective Decision Making
}

\author{
Laura A. Rafferty \\ Southampton University \\ L.Rafferty@soton.ac.uk
}

\author{
Neville A. Stanton \\ Southampton University \\ N.Stanton@soton.ac.uk
}

\author{
Guy H. Walker \\ Southampton University \\ G.Walker@soton.ac.uk
}

\begin{abstract}
Motivation - To explore the applicability of a Human Factors methodology for the investigation of fratricide. Research approach - The EAST methodology was used to analyse an incident of fratricide and its ability to explore the Famous Five of Fratricide (F3) model was investigated. Findings/Design - The analysis revealed that EAST was able to provide explicit discussion of the Famous Five of Fratricide (F3) models five causal factors of communication, cooperation, coordination, schemata and situation awareness. Research limitations/Implications - The research explored a single case study and as such is couched at the initial phases of investigation. Originality/Value - The analysis provides a contribution to the knowledge surrounding fratricide both with respect to the novel application of the EAST methodology to an incident of fratricide, and also the causal factors identified by EAST within the fratricide incident. Take away message - The EAST methodology provides an innovative way of exploring causality in incidents of fratricide.
\end{abstract}

\section{Keywords}

Accident Analysis, Fratricide, Systems Theory, Human Error, EAST.

\section{THE INCIDENT}

At the end of the Persian Gulf War a mission called Operation Provide Comfort (OPC) was established. The operation was setup as a multinational humanitarian effort for the Kurdish refugees in the Iraqi hills. One aspect of Operation Provide Comfort was to develop a No-Fly Zone (NFZ) over Northern Iraq, also known by the military as the Tactical Area Of Responsibility (TAOR). The coalition Air Force led this operation, supported by the Army, who led the ground operations in the TAOR. This multinational operation involved American, British, Turkish and French fighter pilots who made daily patrols of the TAOR in order to prevent any Iraqi aircraft entering and threatening the humanitarian relief efforts (USAF, 1994). Three years after the implementation of the TOAR, on April 15, 1994, two U.S. Army Black Hawk (BH) helicopters were shot down by two U.S. Air Force F-15s. All 26 of the Black Hawk passengers were killed, including 15 American citizens, and a further 11 consisting of British, French and Turkish military officers and Kurdish citizens (USAF, 1994). Both the F15s and the Black Hawks were flown by experienced pilots, with electronic identification technology. The incident occurred in clear weather with the support of an AWACS (Airborne Warning and Control System) aircraft for surveillance and control of aircraft within the TAOR (USAF, 1994).

Below a simplified event line of the incident is presented, derived from numerous accounts of the incident, including Leveson (2002).

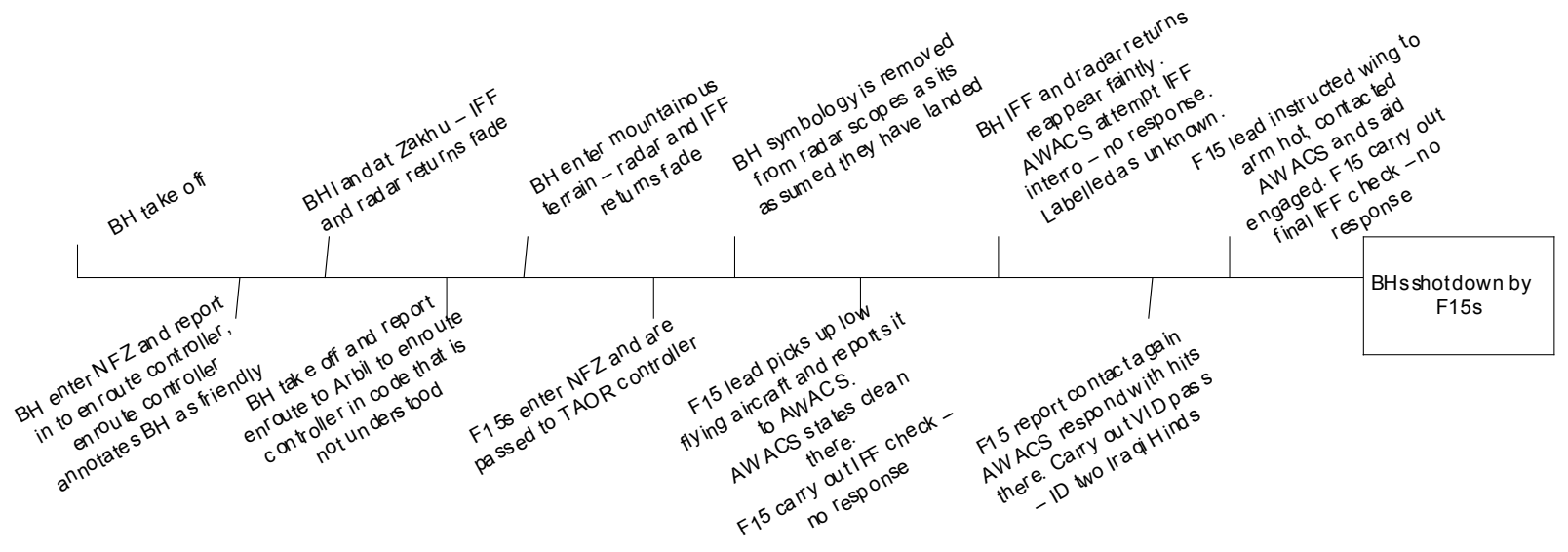

Figure 1 - Simplified event line of incident 


\section{THE EAST METHOD}

The Event Analysis for Systemic Teamwork (EAST) (Baber and Stanton, 2004) provides a systems level methodology that explores emergent properties arising from the interactions within complex, multi agent systems. The EAST methodology consists of the integration of seven individual Ergonomics methods. These methods are: Hierarchical Task Analysis (HTA: Annett, 2005), Coordination Demand Analysis (CDA: Burke, 2005), Communications Usage Diagram (CUD: Watts \& Monk, 2000), Social Network Analysis (SNA: Driskall \& Mullen 2005), Information Networks (IN: e.g. Ogden, 1987) and an enhanced form of Operation Sequence Diagram (OSD: Kirwan \& Ainsworth, 1992). Through the use of multiple methods EAST is able to explore the: who, when, where, what and how of a scenario, as illustrated below in Table 1.

\begin{tabular}{|l|l|l|l|l|l|l|}
\hline & HTA & CDA & CUD & SNA & Info. Nets. & OSD \\
\hline Who & & & & & & \\
\hline When & & & & & & \\
\hline Where & & & & & & \\
\hline What & & & & & & \\
\hline How & & & & & & \\
\hline
\end{tabular}

Table 1 - Methods within EAST methodology

The use of a conglomerate of seven methods validates the results and allows for multiple perspectives within the analysis. Such multiple perspectives are needed in order to explore the emergent properties that arise in complex socio technical systems. Previous applications of EAST (Walker, Gibson, Stanton, Baber, Salmon and Green, 2006; Salmon, Stanton, Walker, Jenkins, Baber and McMaster, 2008) have allowed for a clear illustration of such interplay, depicting the information space in which decisions, and interactions between decision makers took place.

\section{RESULTS AND DISCUSSION}

\section{Hierarchical Task Analysis}

A Hierarchical Task Analysis (HTA) describes a scenario using a hierarchy of goals, sub goals and operations. Every action undertaken within the scenario is broken down in this manner, beginning with the overall goal of the scenario and working down to the individual operations that occurred in order to achieve this goal. An HTA was created for the accident scenario. The HTA provides a representation of the tasks, and the task structures involved in the scenario. This allows for identification of task errors within the scenarios and subsequently identifies possible causal factors in the fratricide incident. The HTA also feeds into many other aspects of the EAST analysis.

\section{Coordination Demands Analysis}

Using the HTA as an input, Coordination Demands Analysis (CDA); (Burke, 2005) can be carried out on the accident scenario. Within any scenario involving teams there are a mixture of team work (tasks that require coordination with others) and task work (tasks that can be performed in isolation) tasks to be undertaken. CDA allows for the division and comparison of these task types. Tasks are extracted from the HTA and rated as either task work tasks or team work tasks. As illustrated in Figure 2 below, the analysis revealed higher levels of teamwork tasks (61\%) than task work tasks (39\%).

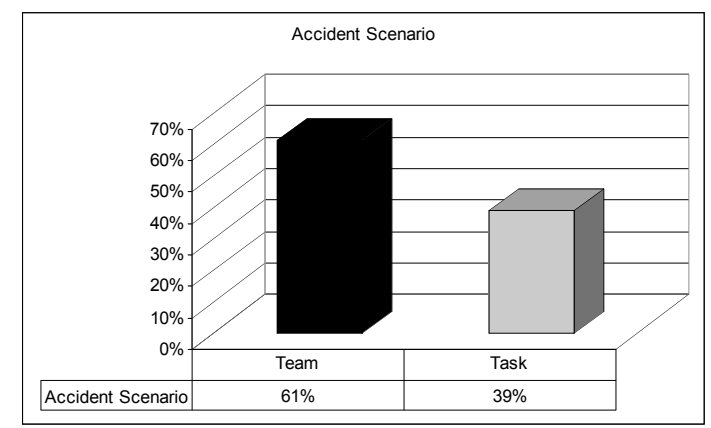

Figure 2 - Team and task work ratios

In this way we can quantitatively measure the level of teamwork that occurred within an accident scenario. Higher levels of teamwork would be hypothesized to act as a preventative measure for fratricide. A high level of task work tasks in the actual scenario may also act as a causal factor in incidents of fratricide. Literature suggests that if the level of work, either task work or team work, becomes too high the workload level may have a negative impact upon the team's performance (Urban, Bowers, Monday \&Morgan, 1995). 
The next stage of the CDA involves the application of a taxonomy containing communication, situation awareness, decision making, mission analysis, leadership, adaptability and assertiveness. This taxonomy is applied to each task step judged to be a teamwork task in the HTA and rated as low, medium or high (1 -3). From the application of this taxonomy a total coordination score can be derived from the mean of the component scores. The scores can be seen below in Figure 3.

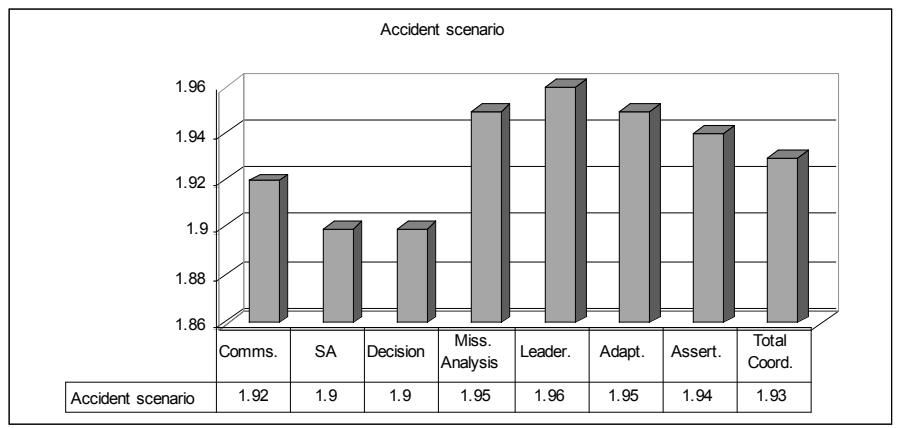

Figure 3 - CDA Analysis

Based upon literature surrounding faulty decision making and resultant accidents (Wiegmann and Shappell, 2001; Reason, 1990) it may be assumed that incidents of fratricide would occur within systems with low levels of coordination activities. The results of the CDA analysis allow this idea to be explored. Overall, the mean total coordination score for the accident scenario is 1.9 (out of a maximum of three). From this it is posited that low levels of coordinated activity may be a causal factor of fratricide. The CDA analysis is able to provide an exploration of coordination. Through the methodology quantitative measurements of coordination can be derived allowing for statistical comparisons between teams or even between scenarios for coordination. In this case low levels of coordination are posited to be a causal factor in incidents of fratricide.

\section{Social Network Analysis}

Social network analysis enables the representation of the communication links between agents in a scenario. The advantage of the network approach is that it focuses on the relationships among agents within their social context (Gibson, Walker, Stanton and Baber, 2004). A social network matrix was developed for the scenario containing all communications that occurred between all agents within the scenario. From this matrix the social network diagram below, Figure 4, can be developed.



Figure 4 - SNA of actual scenario

Examination of the social network reveals the social organisation in the accident scenario. The network appears to be quite convoluted with many actors having links to many other actors and fewer key, or central, actors with fewer, stronger links. From this we can surmise that the accident scenario is quite distributed with a high number of weak communication links. This provides interesting information about the structure of the system.

Once the social network has been created a number of statistics can be derived from it using graph theory (Driskell and Mullen, 2005). These statistics include:

- Sociometric status (represents the contribution an individual makes to overall communication activity, the connectedness (i.e. number of connections to other nodes) of a particular information element). 
- Cohesion (the degree of cohesive bonds - direct connections - between actors)

\section{Sociometric status}

The greater the number of sociometric status an agent has the greater the contribution an agent makes to the communication flow of the network. Figure 5, below illustrates the sociometric status levels for all actors involved in the accident scenario.



Figure 5 - Social network sociometric status

In this way EAST is able to illustrate the individual actors, and the overall systems level of sociometric status, or the level of contribution to communication. From this we can discuss differences in communication between actors, between teams and between scenarios.

\section{Cohesion}

The cohesion metric provides a quantitative measurement of cohesion (another name for cooperation). Within social network analysis cohesion refers to dense ties between members of a network, for example, actor A and B are both linked to actor $\mathrm{C}$, as well as to each other. The cohesion metric for the entire scenario was calculated to be 0.10256 . In this way the social network analysis a quantitative measure of cooperation can be derived, allowing for statistical comparison between teams and between scenarios.

\section{Communication Usage Diagram}

A Communication Usage Diagram was developed for the accident scenario. This involved identifying all communication that occurred during the scenario, from the HTA and other accounts of the incident, and the means through which this communication occurred. The analysis showed that all communication that was through either radio contact, was face to face, or was in paper format. A summary of the CUD results for the scenario are illustrated below in Figure 11.

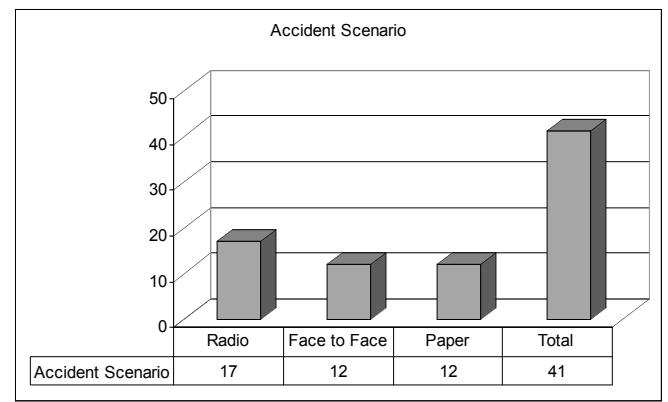

Figure 11 - Summary of ideal and actual scenario CUD

In this way the CUD analysis allows for the identification of the total number of communication acts within an accident scenario. The communication usage diagram allows for a quantitative figure to be placed on the communication occurring within any given scenario, enabling a statistical comparison of communication between teams and between scenarios.

\section{Information Networks}

The EAST methodology involves the construction of Information Networks which in this analysis were formed through close examination of the accident report transcript and from the HTA developed earlier in the EAST analysis. Information Networks consist of a set of nodes that represent information related to the scenario; sources of information and agents. These information objects are linked together through causal pathways, for example, the object [preparation] has the property of [briefing] associated with it, and the object [briefing] has the property of [ATO] associated with it, and so forth, see Figure 13 below. 


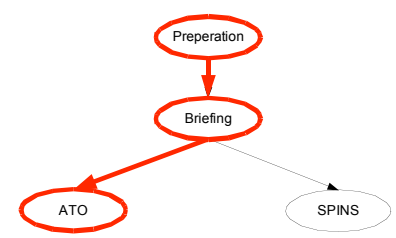

Figure 136 - Example extract from knowledge network

Information Networks allow for the exploration of two of the Famous Five of Fratricide models causal factors: situation awareness and schemata. These concepts are tightly coupled with one another. Neisser's (1976) concept of the perceptual - action cycle clearly illustrates the way in which schema and situation awareness are intertwined. Neisser hypothesises that individuals hold anticipatory schema which direct their attention in any given situation. The individual then interacts with the world and gains situation awareness, based upon these anticipatory schema. The situation awareness then acts to modify the individuals schema based upon this interaction with the world. This modified schema then guides further interaction with the world, which again causes modification of the schema, and so forth in this cyclical manner. Every person's schema will be different based upon their differing life experiences. As we use schemata to drive our information processing they have a huge impact upon which information we process and the way in which we interpret this information. Two people can receive the same information yet process it in entirely different ways due to their differing schemata.

The genotype phenotype distinction (Stanton et al, 2008) presents a useful way of distinguishing between schemata and situation awareness. Genotype (schema) refers to wider systemic factors that influence the development of individual cognitive phenomena and behaviour. Phenotype refers to the local, individual specific manifestation of cognition and behaviour (situation awareness). Schemata represent a generic enduring model of the world that sets expectations and guides search for information, whereas situation awareness represents a more transient, moment by moment flow of current information.

\section{Situation awareness}

The overall information network for the accident scenario represents the systems situation awareness. In order to explore the development of situation awareness and schemata throughout the scenario, the scenario can be broken down into phases. In this case it has been broken down into eleven distinct phases based upon the event line created earlier. Information Networks have been derived for each of these eleven phases. The present discussion will focus upon the key information objects identified. Graph theory metrics, as used in the social network analysis, were employed in order to identify the key information objects as identified as those with a value of the mean + one standard deviation.

Below Table 2 contains a summary of the key information objects used in each phase of the accident scenario.

\begin{tabular}{|l|l|l|l|l|l|l|l|l|l|l|l|}
\hline Information Objects & 08.22 & 09.21 & 09.27 & 09.54 & 10.12 & 10.15 & 10.20 & 10.21 & 10.22 & 10.24 & 10.25 \\
\hline Summarised ROE & & & & & & & & & & & \\
\hline Army Ops Prep & & & & & & & & & & & \\
\hline AWACS mission & & & & & & & & & & & \\
\hline ATO & & & & & & & & & & & \\
\hline Air Ops Prep & & & & & & & & & & & \\
\hline F15 mission & & & & & & & & & & & \\
\hline Air Ops & & & & & & & & & & & \\
\hline ACO & & & & & & & & & & & \\
\hline Army Ops & & & & & & & & & & \\
\hline SITREP & & & & & & & & & & \\
\hline BH mission & & & & & & & & & & & \\
\hline ENROUTE IFF mode & & & & & & & & & & & \\
\hline ENROUTE radio freq. & & & & & & & & & & & \\
\hline AWACS tracking & & & & & & & & & & & \\
\hline F15 report contact & & & & & & & & & & & \\
\hline
\end{tabular}

Table 2 -Key information objects

Table 2 illustrates the development of situational awareness throughout the scenario. The majority of the key information objects remained stable throughout the accident scenario. The information objects AWACS tracking, En- 
route IFF mode and En-route radio freq. were only key information objects during the phases of the scenario in which AWACS were actively tracking the Black Hawk helicopters, who were using the En-route IFF and radio freq. The table also highlights the lack of $B H$ mission as a key knowledge object before the Black Hawks entered the TAOR at 09:21. This leads to the assumption the knowledge of the Black Hawk mission was not disseminated throughout the system before the Black Hawks entered the TAOR.

\section{Individual and team situation awareness}

In order to explore these differences more fully the Information Networks can be broken down to represent individual actors' situation awareness within the scenario. Figure 15 below, illustrates the initial knowledge network (8:22) for the accident scenario. This network has been colour coded with respect to the activation of each information element by each actor. Each actor is assigned a colour and the network is coloured to illustrate, put simply, who knows what.



Figure 15 - 8.22 Information Network.

The ability to derive each individual actor's Information Networks allows us to compare the situation awareness of individual members, and of teams with one another, in the same way as we explore the system level situation awareness. This allows for quantitative measures (for example, sociometric status for key information objects) to be derived and compared across a variety of systemic levels and scenarios. In this way EAST not only explores situation awareness, it explores situation awareness across systemic levels and provides quantitative measures to allow for statistical comparison.

\section{Schemata}

From the identification of individual actors situation awareness an examination of individual actor's schemata can be undertaken. If we focus upon the F15 pilots information elements [those in blue] it can be seen that the pilots were aware that there may be a Black Hawk flight that day, but that all other information they received led them to believe that at the time they were in the TAOR, no other friendly aircraft were. The schema held by the F15 pilots of no friendly aircraft in the area was developed due to the OPC policy that no aircraft were allowed to enter TAOR before the F15 sweep. The OPC policy that no aircraft were allowed to enter without AWACS coverage also meant that when AWACS said they had no information updates for the F15s, the F15s assumed this meant there were no updates regarding friendly forces in the area. In addition to this the daily flight schedule; the F15 briefing; AWACS no contacts there report; AWACS unknown contact their report; IFF interrogation lack of response; incorrect VID pass due to additional fuel tanks all served to reinforce the schema of no friendly aircraft in area. The schema has now been reinforced several times in a short time period, it is now a strong schema and would require substantial negative confirmation to break or change it. This direct negative confirmation did not occur and the schema was maintained, all future information served to reinforce the initial schema of no friendly forces in the area. At each of these points within the scenario the F15 pilots were predisposed to believe that the contact was enemy, that there were no friendly aircraft in the area, and at each of these points the predisposition was strengthened. It is this way that individual actors Information Networks can allow for the exploration of problems at the level of schema present within the accident scenario. 


\begin{tabular}{|l|l|l|l|}
\hline & F15 & AWACS & BH \\
\hline No friendly aircraft in the area & $\checkmark$ & & \\
\hline F15- F16 rivalry & $\checkmark$ & & \\
\hline Helicopters don't fly into TAOR & & $\checkmark$ & \\
\hline IFF returns drop means helicopters landed & & $\checkmark$ & \\
\hline AWACS tracking of helicopters & $\checkmark$ & $\checkmark$ & $\checkmark$ \\
\hline Appropriate radio freq. & & & $\checkmark$ \\
\hline Appropriate IFF mode & & & $\checkmark$ \\
\hline ROE & $\checkmark$ & $\checkmark$ & \\
\hline VID of Black Hawk helicopter & $\checkmark$ & & \\
\hline
\end{tabular}

Table 3 - Key schemata.

Table 3 above highlights the key schemata for the accident scenario. Woods et al (1994) highlight the importance of the activation of knowledge. It is not enough to have knowledge in a system, people must be aware of this knowledge, it must be activated. EAST is able to not only illustrate the knowledge within a system, but through its Information Networks it is able to identify which information objects are activated and by whom. EAST enables the analyst to go even further and identify possible reasons for the activation of information objects through its exploration of schemata. Metrics such as sociometric status allow for the comparison of key information objects and situation awareness between individuals, teams and even systems. This allows for identification of schema and information that may have acted as causal factors within incidents of fratricide. Such analysis enables thorough exploration of two of the five causal factors from the Famous Five of Fratricide model.

\section{Operation Sequence Diagram}

The Operation Sequence Diagram (OSD) presents a graphical illustration of the sequence of events that occurred, who was involved in the incident and the time at which it occurred. The OSD provides a useful graphical representation of a summary of the results of the HTA, CDA, CUD and SNA. Unfortunately the OSD for this scenario is too large to be incorporated into the main body of this paper. Table 4 below represents operations loading table for the accident scenario, one aspect of the OSD.

\begin{tabular}{|l|l|l|l|l|l|}
\hline & Operation & Transmit & Receive & Request & Total \\
\hline Total & 62 & 161 & 161 & 2 & 386 \\
\hline
\end{tabular}

Table 4 - Operations Loading Table

The operation loading table provides a quantitative measure of the amount of communication operations (in comparison to the total number of operations) that occurred during any given scenario. In this case we can see that receive and transmit (communication operations) account for a large amount of the total operations occurring. Within the accident scenario communication accounts for 322 of the 386 operations that occurred. The OSD loading table provides us with a further quantitative measurement of communication that can be used to statistically compare communication between teams and scenarios in order to provide a fuller exploration of one of the Famous Five of Fratricide models causal factors.

\begin{tabular}{|l|l|}
\hline Famous Five & EAST method \\
\hline Communication & $\begin{array}{l}\text { Social Network Analysis } \\
\text { Communication Usage Diagram } \\
\text { Operation Sequence Diagram }\end{array}$ \\
\hline Coordination & $\begin{array}{l}\text { Coordination Demands } \\
\text { Analysis }\end{array}$ \\
\hline Cooperation & Social Network Analysis \\
\hline Schema & Generic Information Networks \\
\hline Situation Awareness & Specific Information Networks \\
\hline
\end{tabular}

Table 5 - EAST methods and Famous Five 


\section{CONCLUSION}

Previous work by the author has identified the need for a complex systemic method for exploration of incidents of fratricide, and to validate the Famous Five of Fratricide model. The EAST methodology was applied to an incident of fratricide in order to ascertain its ability to analyse incidents of fratricide and to validate the Famous Five of Fratricide model. The application of EAST enabled data to be inputted into each section of the model: communication, cooperation, coordination, schemata and situation awareness.

EAST is able, not only, to provide a discussion of all of the Famous Five of Fratricide models causal factors, but is also able to provide quantitative measurements of these factors. This enables statistical comparison of the causal factors between teams and between scenarios and enables a greater depth of exploration of the causal factors behind teamwork breakdowns, specifically here for the investigation of fratricide.

In conclusion, the application of the EAST methodology to an incident of fratricide provided a complex and systemic method that was able to explore the accident scenario thoroughly. The methodology addressed each of the causal factors present within the Famous Five of Fratricide model thus provides a new methodology for the analysis of fratricide incidents and a way in which to validate and build upon the model.

\section{REFERENCES}

Annett, J. (2005). Hierarchical task analysis. In N. A. Stanton et al. (Eds), Handbook of Human Factors and Ergonomics Methods (pp. 33.1-33.7). London: CRC.

Baber, C. and Stanton, N.A. (2004) Methodology for DTC-HFI WP1 field trials. Defence Technology Centre for Human Factors Integration. Report 2.1.

Burke, S. C. (2005). Team Task Analysis. In N. A. Stanton et al. (Eds.), Handbook of Human Factors and Ergonomics Methods (pp. 56.1-56.8). London: CRC.

Driskell, J. E., \& Mullen, B. (2005). Social Network Analysis. In N. A. Stanton et al. (Eds.), Handbook of Human Factors and Ergonomics Methods (pp. 58.1-58.6). London:CRC.

Gibson, H., Walker, G., Stanton, N. And Baber, C. (2004). Report on Results of EAST Methodology for Railway Data: Possession Scenario. HFIDTC/WP1.1.3/7. Version 1.

Kirwan, B. And Ainsworth, L.K. (1992). A guide to Task Analysis. London: Taylor and Francis.

Leveson, N. (2002). A New Approach to System Safety Engineering. Cambridge, MA: Aeronautics and Astronautics, Massachusetts Institute of Technology.

Neisser, U. (1976). Cognition and Reality. San Francisco: Freeman.

Ogden, G.C. (1987). Concept, knowledge and thought. Annual Review of Psychology, 38, 203 - 227.

Salmon, P.M., Stanton, N.A, Walker, G.H., Jenkins, D.P., Baber, C. and McMaster, R. (2008) Representing situation awareness in collaborative systems: A case study in the energy distribution domain. Ergonomics. 51: 3, $367-384$.

Stanton, N. A., Salmon, P. M., Walker, G. H. and Jenkins, D. (2009)'Genotype and phenotype schemata and their role in distributed situation awareness in collaborative systems'. Theoretical Issues in Ergonomics Science,10:1,43 - 68

Urban, J.M., Bowers, C.A., Monday, S.D. and Morgan Jr, B.B. (1995).Workload, Team Structure, and Communication in Team Performance. Military Psychology, 7(2), 123-139.

Walker, G.H., Gibson, H., Stanton, N.A., Baber, C., Salmon, P. and Green, D. (2006). Event analysis of systemic teamwork (EAST): a novel integration of ergonomics methods to analyse C4i activity. Ergonomics, 49:12,1345 1369.

Watts, L.A. and Monk, A.F. (2000). Reasoning about tasks, activities and technology to support collaboration. In Task Analysis, J. Annett and N. Stanton (Eds.), pp 55-78 (London: Taylor and Francis).

Wiegmann, D. A., \& Shappell, S. A. (2001). Human error analysis of commercial aviation accidents: Application of the Human Factors Analysis and Classification System (HFACS). Aviation, Space, and Environmental Medicine, 72, 1006-1016.

USAF Aircraft Accident Investigation Board. U.S. Army Black Hawk Helicopters 87-26000 and 88-26060: Volume 1, Executive Summary: UH-60 Black Hawk Helicopter Accident, 14 April 1994. Available from www.schwabhall.com/opc report.htm. 